\title{
Linear and nonlinear modulus surfaces in stress space, from stress-strain measurements on Berea sandstone
}

\author{
M. Boudjema ${ }^{1}$, I. B. Santos ${ }^{1}$, K. R. McCall ${ }^{1}$, R. A. Guyer ${ }^{2}$, and G. N. Boitnott ${ }^{3}$ \\ ${ }^{1}$ Department of Physics, University of Nevada, Reno, USA \\ ${ }^{2}$ Department of Physics, University of Massachusetts, Amherst, USA \\ ${ }^{3}$ New England Research Incorporated, White River Junction, Vermont, USA
}

Received: 9 January 2002 - Revised: 19 May 2003 - Accepted: 17 July 2003

\begin{abstract}
The elastic response of many rocks to quasistatic stress changes is highly nonlinear and hysteretic, displaying discrete memory. Rocks also display unusual nonlinear response to dynamic stress changes. A model to describe the elastic behavior of rocks and other consolidated materials is called the Preisach-Mayergoyz (PM) space model. In contrast to the traditional analytic approach to stress-strain, the PM space picture establishes a relationship between the quasistatic data and a number density of hysteretic mesoscopic elastic elements in the rock. The number density allows us to make quantitative predictions of dynamic elastic properties. Using the PM space model, we analyze a complex suite of quasistatic stress-strain data taken on Berea sandstone. We predict a dynamic bulk modulus and a dynamic shear modulus surface as a function of mean stress and shear stress. Our predictions for the dynamic moduli compare favorably to moduli derived from time of flight measurements. We derive a set of nonlinear elastic constants and a set of constants that describe the hysteretic behavior of the sandstone.
\end{abstract}

\section{Introduction}

The strain response of rock to quasistatic stress cycles (e.g. stress cycles at $10^{-3} \mathrm{~Hz}$ ) is highly nonlinear and hysteretic (Holcomb, 1981), and displays discrete memory (Guyer et al., 1997). Rocks also display unusual nonlinear behavior in dynamic stress cycles, e.g. acoustic wave experiments at $10^{4} \mathrm{~Hz}$, (Guyer et al., 1999). Nonlinearity and hysteresis are prominent features in the elastic behavior of rocks (Guyer and Johnson, 1999). This observation is the key to making the connection between low frequency (quasistatic) and high frequency (acoustic) measurements, and therefore between the static modulus and the dynamic acoustic velocity of a sample.

Correspondence to: $\mathrm{M}$. Boudjema

(mouna@physics.unr.edu)
A model to describe the elastic behavior of rocks and other consolidated materials is called the Preisach-Mayergoyz (PM) space model (Mayergoyz, 1985; Preisach, 1935). The PM space model uses the statistical properties of many mesoscopic hysteretic elastic elements to describe the elastic response of a macroscopic piece of material (McCall and Guyer, 1996). It allows us to invert quasistatic stress-strain data for the distribution of hysteretic elastic elements. From this distribution, the high frequency acoustic response of the macroscopic piece of material can be predicted.

In this paper, we use the PM space model to analyze quasistatic stress-strain data taken on Berea sandstone. From this analysis we determine the linear elastic constants, the coefficients of cubic nonlinearity, and the coefficient that characterizes the hysteretic response of the rock to compressional and shear disturbances. From the linear elastic constants we find values of the acoustic wave velocities that compare well with experiments on Berea sandstone.

\section{Elastic theory}

Our goal is to deduce something about the nature of the elastic elements in a piece of Berea sandstone from quasistatic stress-strain data. In the context of a suitable theory we can use these deductions to predict the response of the rock to stress protocols different from the ones that were measured. For example, we may predict the response of the rock to dynamic loading in a resonant bar experiment or in an acoustic wave experiment. Detailed understanding of the behavior of the elastic elements may expand our understanding of their microscopic character and the microscopic mechanism for the elastic hysteresis. However, in order to let the data be our guide, we proceed without imposing a prior model of the mechanism for nonlinearity and hysteresis.

\subsection{Linear elasticity}

Consider an isotropic homogeneous elastic system, whose behavior is characterized by two constants. Unlike a liquid 
characterized by one elastic constant, a solid has the means to transform forces in one direction into forces in the perpendicular direction. The magnitude of this transformation, the Poisson's ratio, is an important component of the description of the solid. A solid with a very small Poisson's ratio transforms almost no force from one direction to the perpendicular direction. In terms of the Young's modulus $E$, and the Poisson's ratio $v$, the diagonal elements of the strain field in the solid are

$\epsilon_{x x}=\frac{1}{E}\left[\sigma_{x x}-v\left(\sigma_{y y}+\sigma_{z z}\right)\right]$,

$\epsilon_{y y}=\frac{1}{E}\left[\sigma_{y y}-v\left(\sigma_{x x}+\sigma_{z z}\right)\right]$,

$\epsilon_{z z}=\frac{1}{E}\left[\sigma_{z z}-v\left(\sigma_{x x}+\sigma_{y y}\right)\right]$,

where $\epsilon_{i j}\left(\sigma_{i j}\right)$ is the $j$ th component of the strain (stress) in the $i$ th direction. These equations state that the strain in a single direction results from the response of the system to forces in all directions.

The forces in the interior of an elastic material are not necessarily trivially related to the external forces that are applied to the material. For example, unconsolidated granular materials display internal force chains that have magnitudes not easily related to the external forces. Here we assume that Eqs. (1)-(3) hold for each elastic element within the system.

To simplify Eqs. (1)-(3), we assume that $\sigma_{x x}=\sigma_{y y}$, and rewrite them in terms of mean stress $\sigma$, shear stress $\tau$, volumetric strain $\kappa$, shear strain $\gamma$, bulk modulus $K$, and shear modulus $G$, where these quantities can be defined in terms of our previous quantities:

$$
\begin{aligned}
\sigma & =\frac{1}{3}\left(\sigma_{z z}+2 \sigma_{x x}\right), \\
\tau & =\frac{1}{2}\left(\sigma_{z z}-\sigma_{x x}\right), \\
\kappa & =\epsilon_{z z}+2 \epsilon_{x x}, \\
\gamma & =\epsilon_{z z}-\epsilon_{x x}, \\
K & =\frac{E}{3(1-2 v)}, \\
G & =\frac{E}{2(1+v)} .
\end{aligned}
$$

Equations (1)-(3) reduce to two uncoupled equations,

$\kappa=\frac{\sigma}{K}$,

$\gamma=\frac{\tau}{G}$.

These stress-strain equations are valid for linear elastic systems. They are intuitively appealing, since they imply that given purely diagonal stresses applied in an experiment, the elastic behavior of the system can be understood in terms of two independent elastic coefficients in uncoupled equations.

\subsection{Nonlinear elasticity}

Consolidated materials are elastically nonlinear. Thus, Eqs. (10) and (11) are not strictly valid. The traditional

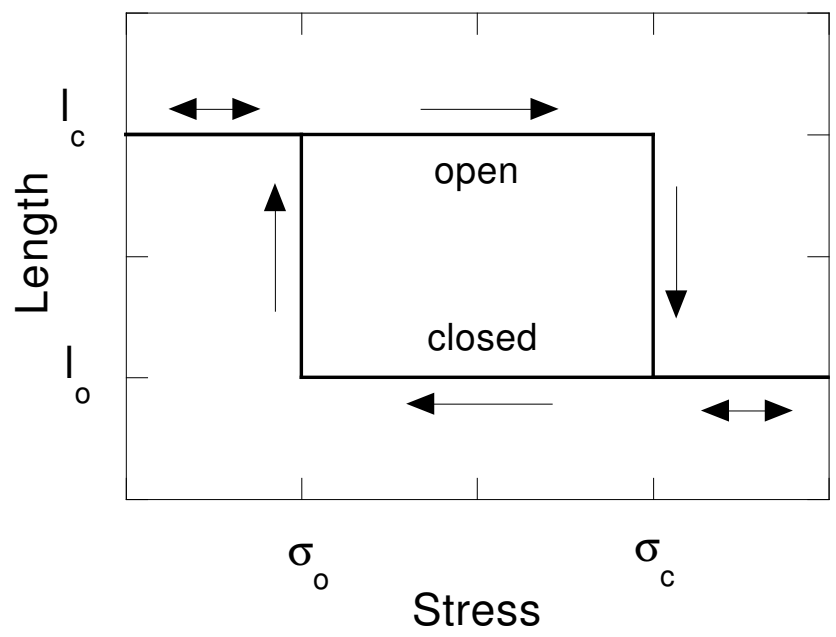

Fig. 1. Hysteretic elastic element. In the PM space model, the elastic properties of a macroscopic system are due to an ensemble of hysteretic elastic elements. Each element has an equilibrium length that switches between two states hysteretically.

model of nonlinear elasticity includes third order strain terms in the energy density of a solid body (Landau and Lifshitz, 1959), and introduces three extra moduli, $A, B$, and $C$. (Here we have defined strain as a linear quantity, $\epsilon_{i j}=\partial u_{i} / \partial x_{j}$, where $u_{i}$ is the $i$ th component of the displacement, and $x_{j}$ is the $j$ th component of the coordinate system.) Given the energy density $\mathcal{E}$, each component of stress is given by $\sigma_{i j}=\partial \mathcal{E} / \partial\left(\partial u_{i} / \partial x_{j}\right)$.

A very complicated set of equations can be simplified by considering the constraints on our system, i.e. only diagonal stresses, and $\sigma_{x x}=\sigma_{y y}$. In our case, the number of stress and strain variables can be reduced to four, and we find

$$
\begin{aligned}
\sigma=K \kappa & +\left(\frac{K}{2}+\frac{A}{9}+B+C\right) \kappa^{2} \\
& +\frac{1}{3}\left(K+\frac{4 G}{3}+\frac{2 A}{3}+2 B\right) \gamma^{2}, \\
\tau=G \gamma & +\left(\frac{K}{2}+\frac{2 G}{3}+\frac{A}{3}+B\right) \gamma \kappa \\
& +\frac{1}{2}\left(G+\frac{A}{3}\right) \gamma^{2} .
\end{aligned}
$$

Equations (12) and (13) can be inverted to find strain as a function of stress, the analogs of Eqs. (10) and (11). To second order in the stresses,

$$
\begin{gathered}
\kappa=\frac{1}{K} \sigma-\frac{T_{1}}{2} \sigma^{2}-\frac{2 T_{2}}{3} \tau^{2}, \\
\gamma=\frac{1}{G} \tau-T_{2} \sigma \tau-\frac{T_{3}}{2} \tau^{2},
\end{gathered}
$$

where

$$
T_{1}=\frac{1}{K^{3}}\left(K+\frac{2 A}{9}+2 B+2 C\right),
$$




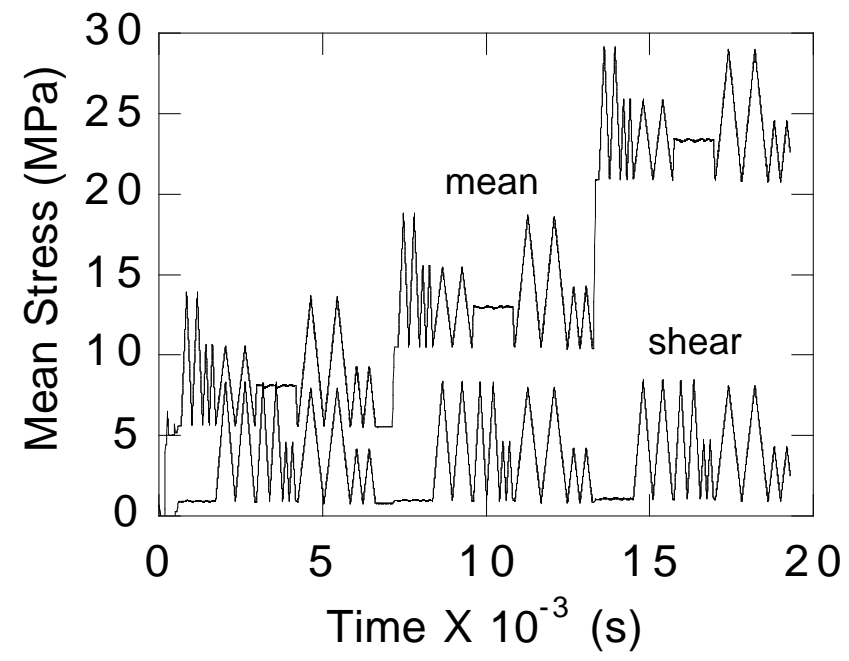

Fig. 2. Stress Protocol. Experimentally applied shear stress and mean stress as a function of time.

$T_{2}=\frac{1}{K G^{2}}\left(\frac{K}{2}+\frac{2 G}{3}+\frac{A}{3}+B\right)$,

$T_{3}=\frac{1}{G^{3}}\left(G+\frac{A}{3}\right)$.

Once again, Eqs. (14) and (15) are intuitively appealing. These two equations reduce to Eqs. (10) and (11) for small stresses, and deviate from the linear relationships quadratically in stress. Note that the resulting equations are no longer decoupled, i.e. the volumetric strain depends on both shear and mean stress. However, only the shear strain contains a cross term in shear and mean stress.

\subsection{Hysteretic elasticity}

The nonlinear elastic equations, Eqs. (14) and (15), do not have the ability to describe hysteresis, which is prevalent in the elastic response of most rocks and other consolidated materials. Thus, we postulate that the rock responds as if there are two systems of elastic elements, one system responding hysteretically to the mean stress, and one system responding hysteretically to the shear stress. We employ a generalization of the PM space model introduced earlier in the context of uniaxial stress (McCall and Guyer, 1996), to rewrite Eqs. (10) and (11),

$\kappa=\kappa_{0} F(\sigma)$,

$\gamma=\gamma_{0} J(\tau)$

where $\gamma_{0}$ and $\kappa_{0}$ are constants determined from experimental data. The functions $F(\sigma)$ and $J(\tau)$ are integrals over densities of elastic elements in mean stress space and shear stress space respectively. It is these functions that we want to find from experimental data.

The principle involved in the analysis has been previously described and illustrated (Guyer et al., 1997). For example, the mean stress - volumetric strain properties of a macroscopic rock sample are taken to be the result of an ensemble

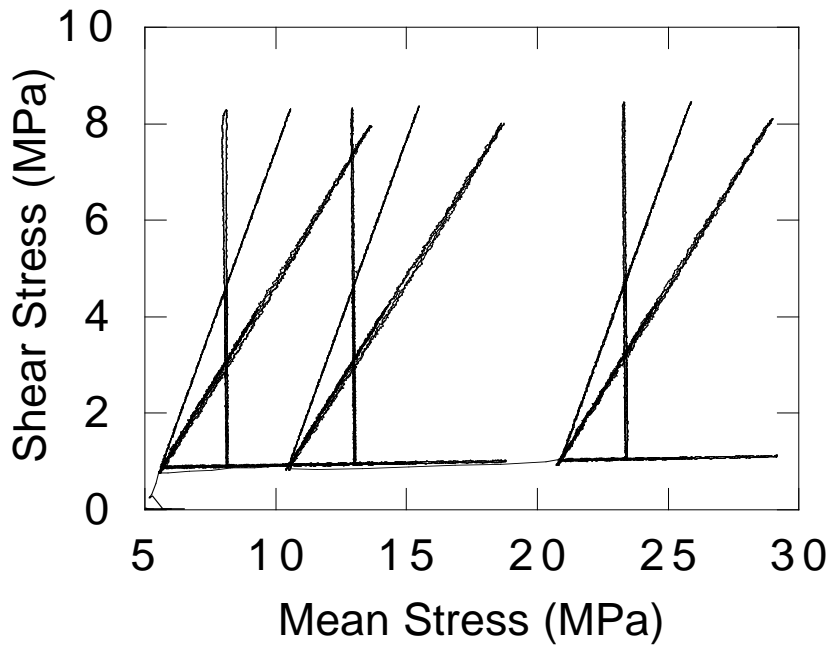

Fig. 3. Stress-Stress Protocol. Applied shear stress as a function of applied mean stress during the experiment. From vertical to horizontal, the protocols are for constant $\sigma, \theta, \mu$, and $\tau$ respectively.

of hysteretic elastic elements with the elastic response illustrated in Fig. 1. Each elastic element is modeled as having an equilibrium length that switches between two states hysteretically. The equilibrium lengths are taken to be equivalent for all elastic elements, thus each element is characterized by two stresses, $\sigma_{c}$ (stress to close) and $\sigma_{o}$ (stress to open).

The volumetric strain response of a macroscopic sample is characterized by a number density of elastic elements $\rho_{\kappa}$ in the space $\left(\sigma_{c}, \sigma_{o}\right) . \quad F(\sigma)$ is given by an integral over $\rho_{\kappa}\left(\sigma_{c}, \sigma_{o}\right)$, where the limits of integration depend on the history of the mean stress protocol. For a closed loop $\sigma_{1} \rightarrow$ $\sigma_{2} \rightarrow \sigma_{1}, F$ is the fraction of elastic elements in $\left(\sigma_{c}, \sigma_{o}\right)$ space that are in the closed state. As the mean stress is increased

$F(\sigma)=\int_{\sigma_{1}}^{\sigma} d \sigma_{c} \int_{\sigma_{1}}^{\sigma_{c}} d \sigma_{o} \rho_{\kappa}\left(\sigma_{c}, \sigma_{o}\right)$.

As the mean stress is decreased,

$F(\sigma)=F\left(\sigma_{2}\right)-\int_{\sigma}^{\sigma_{2}} d \sigma_{c} \int_{\sigma}^{\sigma_{c}} d \sigma_{o} \rho_{\kappa}\left(\sigma_{c}, \sigma_{o}\right)$.

We demand that $F$ be a fraction by norming $F$ to unity over the full $\left(\sigma_{c}, \sigma_{o}\right)$-space; this sets the constant $\kappa_{0}$.

Similarly, $J(\tau)$ is related to the density of elastic elements $\rho_{\gamma}$, in the space $\left(\tau_{c}, \tau_{o}\right)$. These elastic elements produce a shear strain response that depends upon the shear stress history. For a closed loop $\tau_{1} \rightarrow \tau_{2} \rightarrow \tau_{1}, J$ is the fraction of elastic elements in $\left(\tau_{c}, \tau_{o}\right)$-space that are in the closed state. As the shear stress is increased,

$J(\tau)=\int_{\tau_{1}}^{\tau} d \tau_{c} \int_{\tau_{1}}^{\tau_{c}} d \tau_{o} \rho_{\gamma}\left(\tau_{c}, \tau_{o}\right)$

As the shear stress is decreased,

$J(\tau)=J\left(\tau_{2}\right)-\int_{\tau}^{\tau_{2}} d \tau_{c} \int_{\tau}^{\tau_{c}} d \tau_{o} \rho_{\gamma}\left(\tau_{c}, \tau_{o}\right)$. 


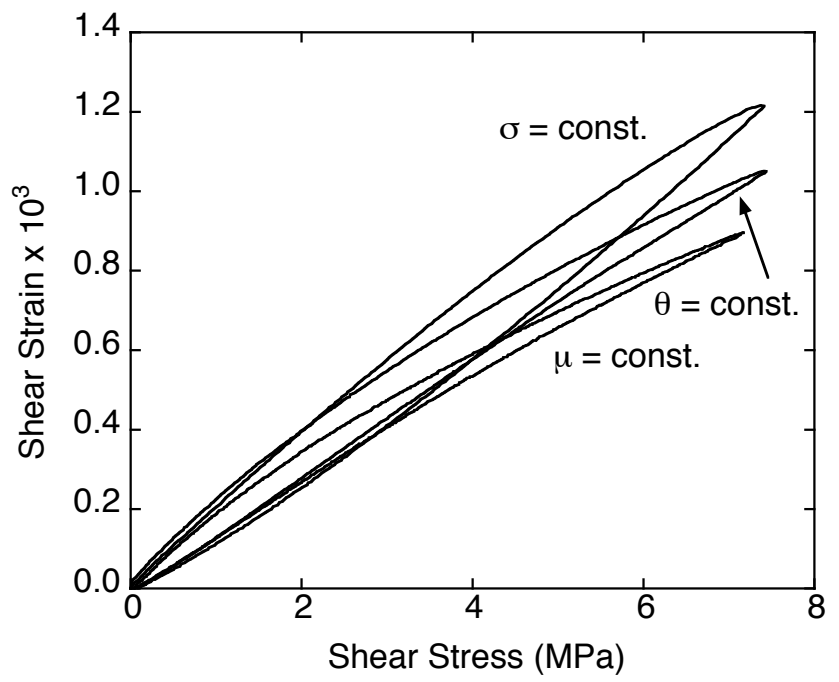

Fig. 4. Shear stress-strain. Shear strain versus shear stress for three protocols at $5 \mathrm{MPa}$ base mean stress (loops $5 \theta 2,5 \sigma 2,5 \mu 2$ ).

$J$ is normed to unity over the full $\left(\sigma_{c}, \sigma_{o}\right)$-space, setting the constant $\gamma_{0}$.

In the limit that the densities $\rho_{\kappa}\left(\sigma_{c}, \sigma_{o}\right)$ and $\rho_{\gamma}\left(\tau_{c}, \tau_{o}\right)$ are diagonal, e.g. $\rho_{\kappa}\left(\sigma_{c}, \sigma_{o}\right)=\chi\left(\sigma_{c}, \sigma_{o}\right) \delta\left(\sigma_{c}-\sigma_{o}\right), F(\sigma)$ and $J(\tau)$ are functions of $\sigma$ and $\tau$ that coincide with Eqs. (14) and (15) for proper choice of $\chi$.

Our goal is to use experimental data to determine $\rho_{\kappa}\left(\sigma_{c}, \sigma_{o}\right)$ and $\rho_{\gamma}\left(\tau_{c}, \tau_{o}\right)$. These number densities can then be used to predict the elastic response of a system under conditions that have not been measured. As the number densities characterize ensembles of elastic elements, they can also be used to infer the important microscopic characteristics of the rock.

\section{Stress-strain experiment}

The data analyzed in this paper are from measurements of axial and radial strain as the sample is subjected to complicated stress protocols (Boitnott, 1997). The measurements were performed in a hydraulically servo-controlled triaxial apparatus with a precision internal load cell in the loading column. The sample was a "room dry" Berea sandstone cored perpendicular to bedding, $44.4 \mathrm{~mm}$ in diameter with length equal to twice the diameter. It was jacketed in $0.127 \mathrm{~mm}$ thick copper foil and instrumented with standard polymide backed constantan foil strain gauges. The axial and radial strains $\left(\epsilon_{z z}\right.$ and $\left.\epsilon_{x x}\right)$ and the axial and radial stresses $\left(\sigma_{z z}\right.$ and $\left.\sigma_{x x}\right)$ were measured. The effects of pressure on the strain gauges were removed using calibrations derived from identically instrumented samples of optical glass for which the elastic constants are known.

The samples were subjected to an elaborate stress protocol involving a limited stress range, $|\sigma| \leq 30 \mathrm{MPa}$, and repeated stress loops so that loops with negligible creep are obtained. The applied stresses and the strain response can be charac-

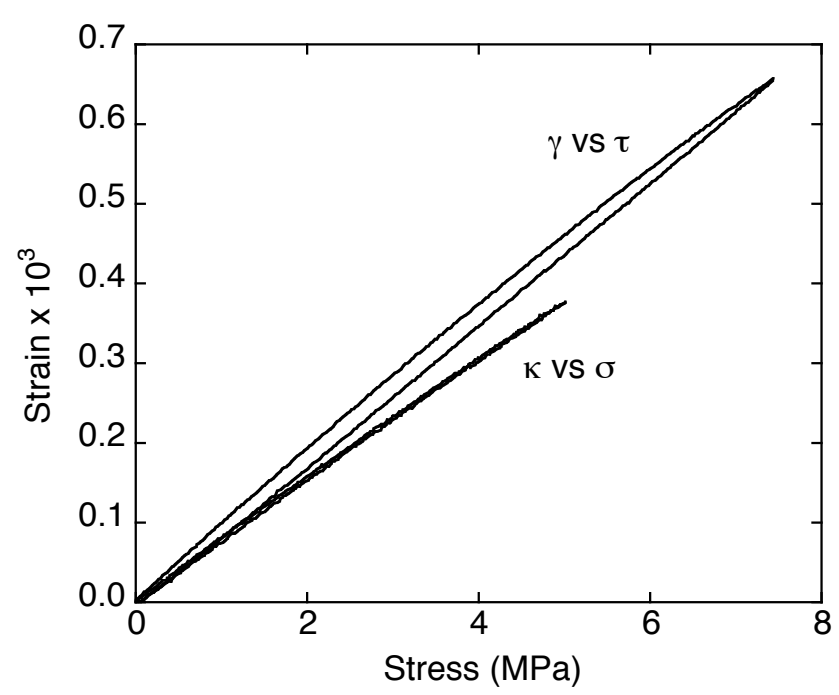

Fig. 5. Stress-strain at constant radial stress. Shear strain versus shear stress, and volumetric strain versus mean stress for loop $20 \theta 2$.

terized by mean stress $\sigma$, shear stress $\tau$, volumetric strain $\kappa$, and shear strain $\gamma$, as defined in Eqs. (4)-(7). At each of three base mean stresses, $5 \mathrm{MPa}, 10 \mathrm{MPa}$, and $20 \mathrm{MPa}$, the samples were subjected to stress loops as the following were held constant: (1) shear stress, (2) radial stress, (3) mean stress, and (4) radial strain. In Fig. 2 we show the stress protocol as a function of time. In Fig. 3 we show the stress protocol in stress space, with coordinates $\sigma$ and $\tau$. From Fig. 2 we see that each scan in stress-strain space has been carried out at least twice. Because the elastic response of rocks has discrete memory the first exploration of any region of stressstrain space is different from all subsequent explorations.

Our analysis will focus on a subset of the complete data set. In particular, we are interested in closed stress loops, $\sigma_{1} \rightarrow \sigma_{2} \rightarrow \sigma_{1}$. We will specify the stress loop under discussion by a sequence of a number, a letter, and a number, e.g. stress loop $20 \sigma 2$. The first number refers to the base mean stress; 5,10 or $20 \mathrm{MPa}$. The letter corresponds to the type of stress loop; $\tau=$ constant shear stress, $\theta=$ constant radial stress, $\sigma=$ constant mean stress, and $\mu=$ constant radial strain. The third number corresponds to the loop number at fixed base mean stress and stress protocol. Thus the loop $20 \theta 2$ is the second loop at constant radial stress, at $20 \mathrm{MPa}$ base mean stress.

There are 30 mean stress and 30 shear stress loops in the complete data set. We will confine our attention to 9 mean stress and 9 shear stress loops, the second loop of each stress protocol, for each base mean stress state. In Fig. 4 we show three shear strain versus shear stress loops, loops $5 \theta 2,5 \sigma 2$, and $5 \mu 2$ (note there is no shear strain loop $5 \tau 2$ ). The stress and strain for each loop have been shifted by a constant amount so that each loop starts and stops at $(\tau, \gamma)=(0,0)$. The strain for increasing stress is always less than the strain for decreasing stress, i.e. the hysteresis loops are always traversed counter clockwise. The stress protocol was chosen 


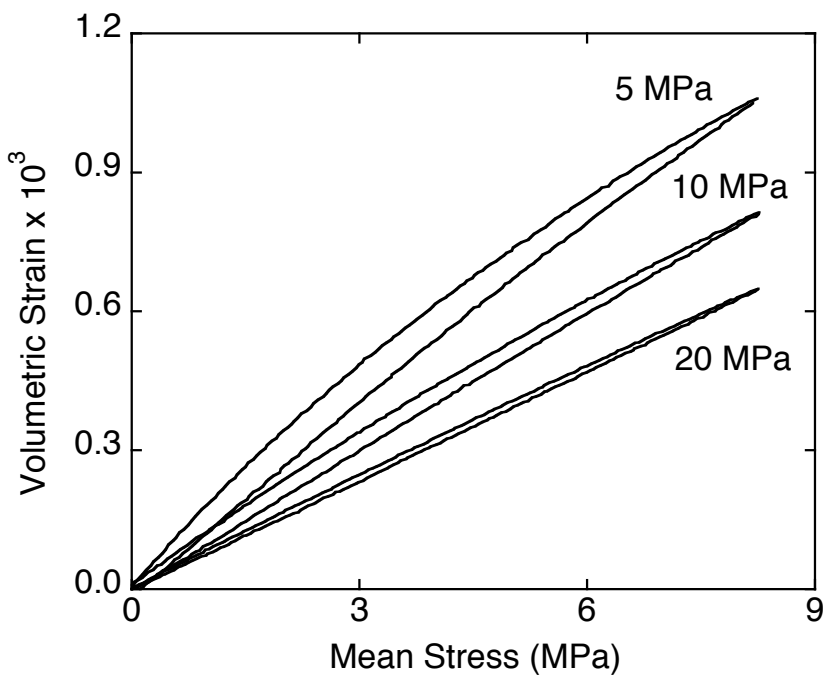

Fig. 6. Volumetric strain - mean stress for $\tau$ constant. Volumetric strain versus mean stress at constant shear stress, for the three base mean stresses (loops $5 \tau 2,10 \tau 2,20 \tau 2$ ).

so that the shear stress change was nominally equivalent for each of these loops. Note that the amount of hysteresis in the $5 \mu 2$ loop is substantially less than that in the $5 \sigma 2$ loop, i.e. the hysteresis is least when the mean stress varies the most, and most when the mean stress is constant.

In Fig. 5 we show the volumetric strain as a function of mean stress, and the shear strain as a function of shear stress for a single experimental loop, 2002. As above, we have shifted stress and strain so that the curves begin at the origin. The two loops differ primarily in that the shear strain shows substantial hysteresis while the volumetic strain has almost none. In Fig. 6 we show volumetric strain versus mean stress at fixed shear stress, for the three base mean stresses, loops $5 \tau 2,10 \tau 2$, and $20 \tau 2$. Note that as the mean stress increases the amount of hysteresis in the volumetric strain decreases. In Fig. 7 we show volumetric strain versus mean stress at fixed radial stress, for the three base mean stresses, loops $5 \theta 2,10 \theta 2,20 \theta 2$. At constant radial stress there is very modest hysteresis in the volumetric strain. Compare this to the hysteresis in the shear strain in Fig. 5.

\section{Analysis}

In this section, we describe analysis of the data using the PM space model. We use a simple analytic model for the number densities, fit the stress-strain data to appropriate functions, and use the results to predict the dynamic moduli, $K_{\mathrm{dyn}}$ and $G_{\text {dyn }}$, in the mean stress - shear stress space of the experiment. Measurements of the compressional and shear velocities on the sample (Boitnott, 1997) yielded values of the dynamic moduli which we compare to the $K_{\mathrm{dyn}}$ and $G_{\mathrm{dyn}}$ surfaces derived from quasistatic data.

Based on observations of the stress-strain data, we argue for a simple model for the number density of elastic units.

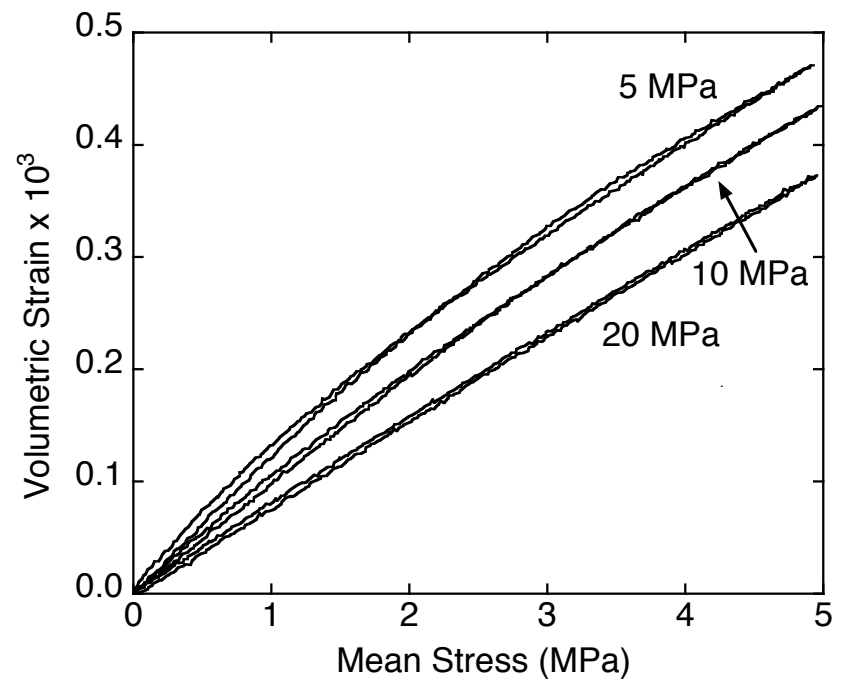

Fig. 7. Volumetric strain - mean stress for $\theta$ constant. Volumetric strain versus mean stress at constant radial stress, for the three base mean stresses (loops 5 $02,10 \theta 2,20 \theta 2$ ).

These observations are: 1 . The hysteresis in stress-strain is obvious, but not enormous. Thus, the number densities should be high on the diagonals $\sigma_{c}=\sigma_{o}$, and $\tau_{c}=\tau_{o}$. Diagonal elastic elements have no hysteresis, as shown in Fig. 1. 2. Nonlinearity in stress-strain decreases as the stresses are increased. Given these two observations, we propose analytic forms for the number densities,

$\rho_{\kappa}\left(\sigma_{c}, \sigma_{o}\right)=\left(D_{\kappa}+H_{\kappa} \sigma_{c}\right) \delta\left(\sigma_{o}-\sigma_{c}\right)+\alpha_{\kappa}$,

and

$\rho_{\gamma}\left(\tau_{c}, \tau_{o}\right)=\left(D_{\gamma}+H_{\gamma} \tau_{c}\right) \delta\left(\tau_{o}-\tau_{c}\right)+\alpha_{\gamma}$.

In Eqs. (25) and (26), the number density is taken to vary linearly with stress on the diagonal, and to have a (small) constant background that accounts for the hysteresis (offdiagonal elements). Since the nonlinearity decreases with increasing stress, we expect $H$ to be negative.

Given the model for number density in Eqs. (25) and (26), the strains $\kappa$ and $\gamma$ can be calculated for closed stress loops using Eqs. (19)-(24). For a closed loop $\sigma_{1} \rightarrow \sigma_{2} \rightarrow \sigma_{1}$ $\left(\kappa_{1} \rightarrow \kappa_{2} \rightarrow \kappa_{1}\right)$, the strain as stress is increasing from $\sigma_{1}$ to $\sigma_{2}$ is given by

$$
\begin{aligned}
\kappa_{\mathrm{up}}(\sigma) & =\kappa_{1}+\kappa_{0}\left[\left(\frac{1}{2}\left(\alpha_{\kappa}-H_{\kappa}\right) \sigma_{1}^{2}-D_{\kappa} \sigma_{1}\right)\right. \\
& \left.+\left(D_{\kappa}-\alpha_{\kappa} \sigma_{1}\right) \sigma+\frac{1}{2}\left(\alpha_{\kappa}+H_{\kappa}\right) \sigma^{2}\right],
\end{aligned}
$$

and the strain as stress is decreasing from $\sigma_{2}$ to $\sigma_{1}$ is given by

$$
\begin{aligned}
\kappa_{\mathrm{dn}}(\sigma) & =\kappa_{2}-\kappa_{0}\left[\left(\frac{1}{2}\left(\alpha_{\kappa}+H_{\kappa}\right) \sigma_{2}^{2}+D_{\kappa} \sigma_{2}\right)\right. \\
& \left.-\left(D_{\kappa}+\alpha_{\kappa} \sigma_{2}\right) \sigma+\frac{1}{2}\left(\alpha_{\kappa}-H_{\kappa}\right) \sigma^{2}\right] .
\end{aligned}
$$




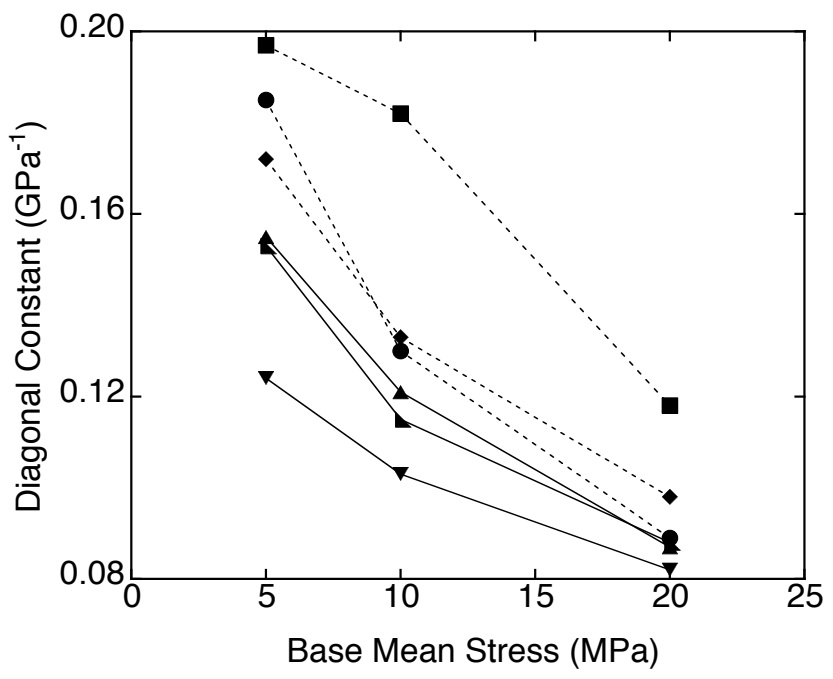

Fig. 8. The diagonal constants of the number density $\kappa_{0} D_{\kappa}$ and $\gamma_{0} D_{\gamma}$. The squares, circles, and diamonds connected by dotted lines are $\kappa_{0} D_{\kappa}$, for $\theta, \tau$, and $\mu$ constant respectively. The triangles connected by solid lines are $\gamma_{0} D_{\gamma}$ : triangles pointing up for $\theta$ constant, right triangles for $\mu$ constant, and triangles pointing down for $\sigma$ constant.

Similarly, for a closed loop $\tau_{1} \rightarrow \tau_{2} \rightarrow \tau_{1}\left(\gamma_{1} \rightarrow \gamma_{2} \rightarrow \gamma_{1}\right)$, the strain as stress is increasing from $\tau_{1}$ to $\tau_{2}$ is given by

$$
\begin{aligned}
\gamma_{\text {up }}(\tau) & =\gamma_{1}+\gamma_{0}\left[\left(\frac{1}{2}\left(\alpha_{\gamma}-H_{\gamma}\right) \tau_{1}^{2}-D_{\gamma} \tau_{1}\right)\right. \\
& \left.+\left(D_{\gamma}-\alpha_{\gamma} \tau_{1}\right) \tau+\frac{1}{2}\left(\alpha_{\gamma}+H_{\gamma}\right) \tau^{2}\right]
\end{aligned}
$$

and the strain as stress is decreasing from $\tau_{2}$ to $\tau_{1}$ is given by

$$
\begin{aligned}
\gamma_{\mathrm{dn}}(\tau) & =\gamma_{2}-\gamma_{0}\left[\left(\frac{1}{2}\left(\alpha_{\gamma}+H_{\gamma}\right) \tau_{2}^{2}+D_{\gamma} \tau_{2}\right)\right. \\
& \left.-\left(D_{\gamma}+\alpha_{\gamma} \tau_{2}\right) \tau+\frac{1}{2}\left(\alpha_{\gamma}-H_{\gamma}\right) \tau^{2}\right] .
\end{aligned}
$$

Thus, by fitting the experimental strain to a quadratic equation in stress, we can find the parameters $D, H$, and $\alpha$ that characterize the number density of the PM space model. That is, if the experimental data can be characterized by the equations

$\kappa_{\text {up }}(\sigma)=a_{0}+a_{1} \sigma+a_{2} \sigma^{2}$,

$\kappa_{\mathrm{dn}}(\sigma)=b_{0}+b_{1} \sigma+b_{2} \sigma^{2}$,

then

$$
\begin{aligned}
\kappa_{0} D_{\kappa} & =\frac{1}{2}\left[a_{1}+b_{1}+\left(\sigma_{2}-\sigma_{1}\right)\left(b_{2}-a_{2}\right)\right], \\
\kappa_{0} H_{\kappa} & =a_{2}+b_{2}, \\
\kappa_{0} \alpha_{\kappa} & =a_{2}-b_{2} .
\end{aligned}
$$

Similar equations hold for the shear stress versus shear strain loops.

One of our goals is to relate quasistatic stress-strain data to dynamic moduli, and thus to acoustic velocities. Recall

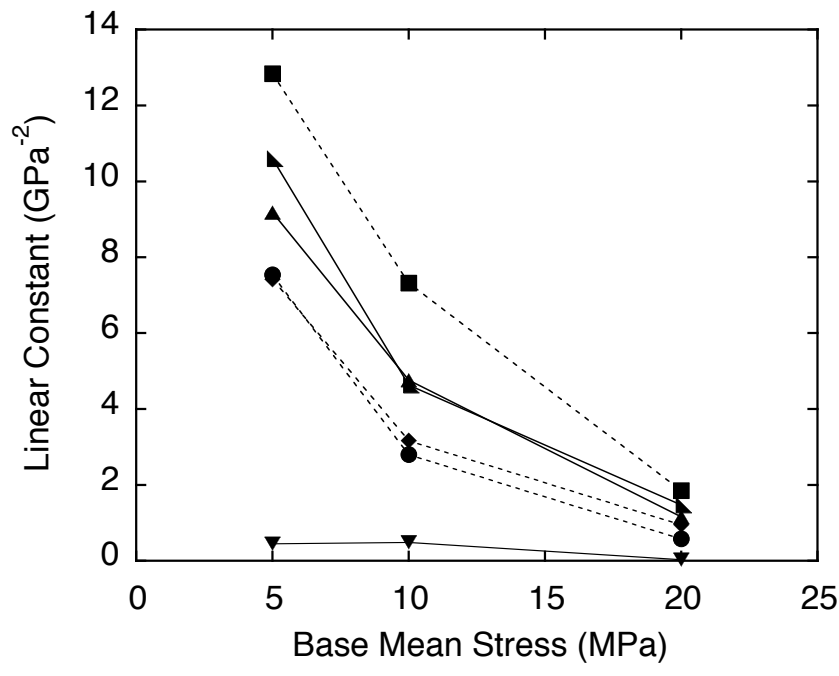

Fig. 9. The diagonal constants of the number density $\kappa_{0}\left|H_{K}\right|$ and $\gamma_{0}\left|H_{\gamma}\right|$. The squares, circles, and diamonds connected by dotted lines are $\kappa_{0}\left|H_{\kappa}\right|$, for $\theta, \tau$, and $\mu$ constant respectively. The triangles connected by solid lines are $\gamma_{0}\left|H_{\gamma}\right|$ : triangles pointing up for $\theta$ constant, right triangles for $\mu$ constant, and triangles pointing down for $\sigma$ constant.

that a propagating wave is just a very small stress-strain loop. Letting $\sigma_{1}=\bar{\sigma}, \sigma=\bar{\sigma}+\delta \sigma$, and $\kappa-\kappa_{1}=\delta \kappa$ in Eq. (27) (or equivalently, letting $\sigma_{2}=\bar{\sigma}, \sigma=\bar{\sigma}-\delta \sigma$, and $\kappa_{2}-\kappa=\delta \kappa$ in Eq. 28), we find

$$
\frac{\delta \kappa}{\delta \sigma}=\frac{1}{K_{\mathrm{dyn}}}=\kappa_{0}\left(D_{\kappa}+H_{\kappa} \bar{\sigma}\right) .
$$

That is, the dynamic bulk modulus depends only on the diagonal part of the number density characterizing the volumetric stress space. A similar calculation for the shear modulus yields

$\frac{\delta \gamma}{\delta \tau}=\frac{1}{G_{\text {dyn }}}=\gamma_{0}\left(D_{\gamma}+H_{\gamma} \bar{\tau}\right)$.

$K_{\text {dyn }}^{-1}$ is explicitly dependent only on $\sigma$, and $G_{\text {dyn }}^{-1}$ is explicitly dependent only on $\tau$. However, each loop from which the moduli are determined follows a trajectory in the twodimensional space of $(\sigma, \tau)$. Thus the moduli are implicitly dependent on both stresses.

We can compare our results to the traditional model of nonlinear elasticity by noting

$\frac{1}{K_{\text {dyn }}}=\frac{d \kappa}{d \sigma}=\frac{\partial \kappa}{\partial \sigma}+\frac{\partial \kappa}{\partial \tau} \frac{\partial \tau}{\partial \sigma}$,

and similarly for $G_{\mathrm{dyn}}^{-1}$. The dynamic moduli from traditional nonlinear elasticity theory are determined by derivatives of Eqs. (14) and (15),

$$
\begin{aligned}
& \frac{1}{K_{\text {dyn }}}=\frac{1}{K}-T_{1} \sigma-\frac{4}{3} T_{2} \frac{\partial \tau}{\partial \sigma} \tau, \\
& \frac{1}{G_{\text {dyn }}}=\frac{1}{G}-T_{2}\left(\sigma+\frac{\partial \sigma}{\partial \tau} \tau\right)-T_{3} \tau .
\end{aligned}
$$


Table 1. The values of $D, H$, and $\alpha$ for each of the 18 stress-strain loops

\begin{tabular}{ccccccc}
\hline Loop & $\kappa_{0} D_{\kappa}, \mathrm{GPa}^{-1}$ & $\kappa_{0} H_{\kappa}, \mathrm{GPa}^{-2}$ & $\kappa_{0} \alpha_{\kappa}, \mathrm{GPa}^{-2}$ & $\gamma_{0} D_{\gamma}, \mathrm{GPa}^{-1}$ & $\gamma_{0} H_{\gamma}, \mathrm{GPa}^{-2}$ & $\gamma_{0} \alpha_{\gamma}, \mathrm{GPa}^{-2}$ \\
\hline $5 \tau 2$ & 0.185 & -7.54 & 3.58 & - & - & - \\
$10 \tau 2$ & 0.130 & -2.80 & 1.80 & - & - & - \\
$20 \tau 2$ & 0.089 & -0.58 & 0.74 & - & - & - \\
$5 \theta 2$ & 0.197 & -12.84 & 0.00 & 0.155 & -9.18 & 6.21 \\
$10 \theta 2$ & 0.182 & -7.32 & 0.00 & 0.121 & -4.76 & 3.73 \\
$20 \theta 2$ & 0.118 & -1.85 & 0.00 & 0.087 & -1.16 & 1.64 \\
$5 \sigma 2$ & - & - & - & 0.124 & -0.45 & 11.38 \\
$10 \sigma 2$ & - & - & - & 0.103 & -0.49 & 5.14 \\
$20 \sigma 2$ & - & - & - & 0.082 & -0.03 & 1.72 \\
$5 \mu 2$ & 0.172 & -7.42 & 2.13 & 0.153 & -10.6 & 4.21 \\
$10 \mu 2$ & 0.133 & -3.17 & 0.97 & 0.115 & -4.63 & 2.60 \\
$20 \mu 2$ & 0.098 & -0.95 & 0.42 & 0.088 & -1.47 & 1.45
\end{tabular}

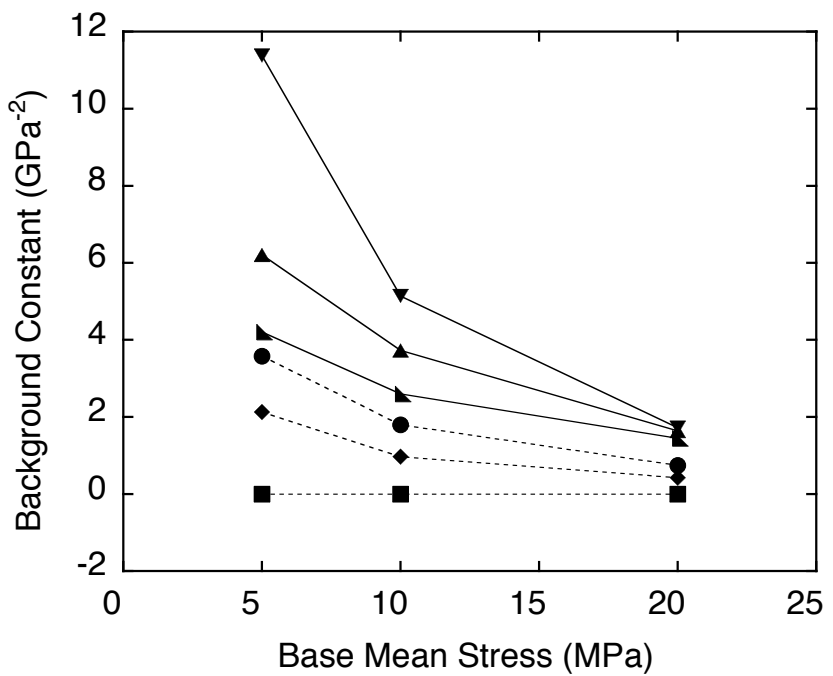

Fig. 10. The off-diagonal constants $\kappa_{0} \alpha_{\kappa}$ and $\gamma_{0} \alpha_{\gamma}$. The squares, circles, and diamonds connected by dotted lines are $\kappa_{0} \alpha_{\kappa}$, for $\theta, \tau$, and $\mu$ constant respectively. The triangles connected by solid lines are $\gamma_{0} \alpha_{\gamma}$ : triangles pointing up for $\theta$ constant, right triangles for $\mu$ constant, and triangles pointing down for $\sigma$ constant.

Analysis of the experimental data will yield $K_{\mathrm{dyn}}^{-1}(\sigma, \tau)$ and $G_{\text {dyn }}^{-1}(\sigma, \tau)$ through Eqs. (36) and (37). The partial derivative $\partial \tau / \partial \sigma$ depends on the stress protocol, and can be determined from the slopes of the $\tau-\sigma$ relations shown in Fig. 3. Thus, the linear and nonlinear moduli $K, G, A, B$, and $C$ can be determined using the experimental data, and Eqs. (39) and (40).

\section{Results}

For each of the 18 stress-strain loops listed in Table 1, 9 covering regions of $\left(\sigma_{c}, \sigma_{o}\right)$-space and 9 covering regions of $\left(\tau_{c}, \tau_{o}\right)$-space, we made quadratic fits to the data (as in

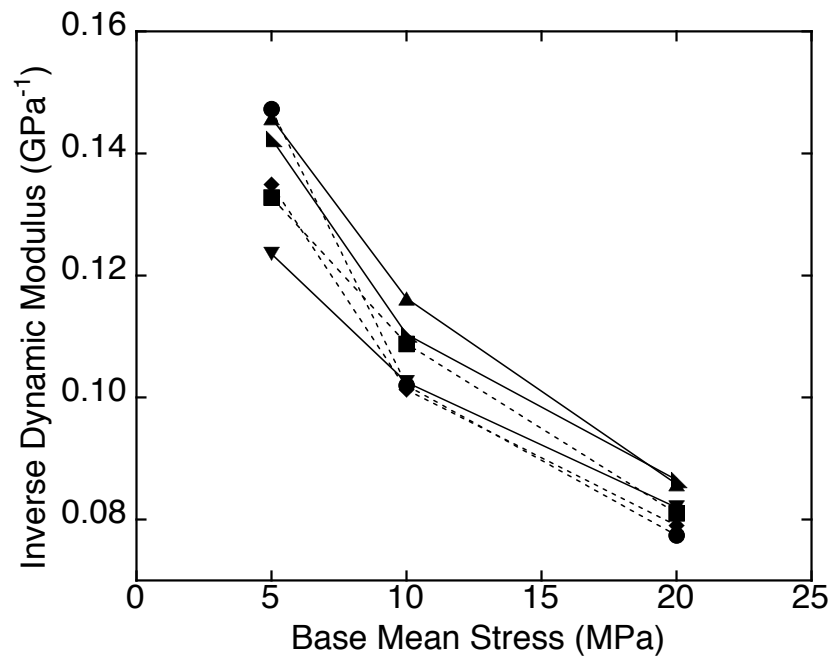

Fig. 11. $K_{\text {dyn }}^{-1}$ and $G_{\text {dyn }}^{-1}$ for $\tau=1 \mathrm{MPa}$. The squares, circles, and diamonds connected by dotted lines are $K_{\mathrm{dyn}}^{-1}$, for $\theta, \tau$, and $\mu$ constant respectively. The triangles connected by solid lines are $G_{\text {dyn }}^{-1}$ : triangles pointing up for $\theta$ constant, right triangles for $\mu$ constant, and triangles pointing down for $\sigma$ constant.

Eqs. 31, 32), and determined the parameters $\kappa_{0} D_{\kappa}, \kappa_{0} H_{\kappa}$, $\kappa_{0} \alpha_{\kappa}, \gamma_{0} D_{\gamma}, \gamma_{0} H_{\gamma}$, and $\gamma_{0} \alpha_{\gamma}$ that characterize the number density (using Eqs. 33-35). These are listed in Table 1. Note that for the constant $\theta$ mean stress loops, $\alpha$ was arbitrarily set to zero. To within the noise in the data, these curves have no hysteresis, as shown in Fig. 7.

Figures 8, 9, and 10 show the values in Table 1 as a function of the base mean stress of the stress-strain loops. As expected, $D,|H|$, and $\alpha$ all decrease as mean stress increases. The moduli $K_{\text {dyn }}$ and $G_{\text {dyn }}$ depend on the inverse combination of $\mathrm{D}$ and $\mathrm{H}$ (Eqs. 36, 37). Thus, the decrease in $D$ indicates that the material is hardening as the base mean stress is increased. The decrease in $|H|$ indicates that the overall curvature of the stress-strain loop decreases as the base mean 


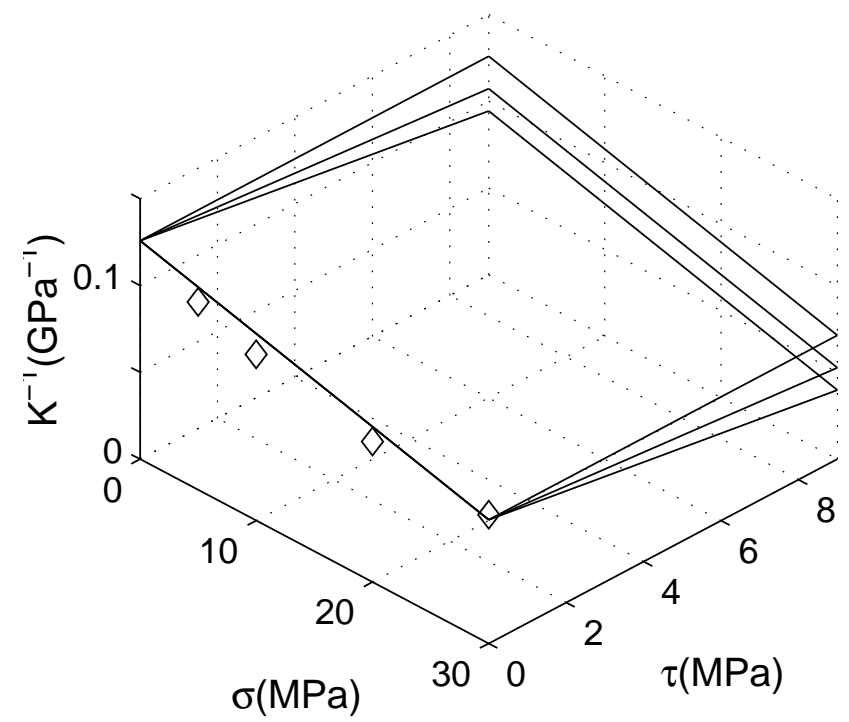

Fig. 12. Planes describing the inverse dynamic bulk modulus. The modulus plane explored is determined by the experimental protocol. In the figure, the top plane describes the modulus for constant $\tau$, the middle plane is for constant $\mu$, and the bottom plane is for constant $\theta$. The diamonds are the result of dynamic measurements as a function of mean stress.

stress is increased. The decrease in $\alpha$ indicates that the hysteresis in the stress-strain loops decreases as the base mean stress is increased.

In Fig. 10, it is clear that shear stress loops are more hysteretic than mean stress loops, i.e. the $\alpha$ 's connected by solid lines are uniformly larger than those connected by dotted lines. Figures 4-7 confirm this point. From a practical standpoint, this means that shear strain will generally be harder to predict than volumetric strain, in the absence of data. Hysteresis is an important component of shear strain response to shear stress changes. The constant $\sigma$ shear stress loops are particularly interesting (see examples in Figs. 4 and 5). They feature small change in $D$ and $H$ (almost no average curvature to a stress-strain loop), and yet very large values of $\alpha$. Thus the average trend of the data is almost linear, but the hysteresis is very large! An explanation can be found in the loops displayed in Figs. 4 and 5, where we see that the curvature of constant $\sigma$ loops changes sign when the applied stress changes direction; thus, the average stress-strain is almost linear, even though the loop is quite fat. In contrast, the constant $\theta$ mean stress loops shown in Fig. 7 have curvature (large values of $D$ and $H$ ), but no hysteresis $(\alpha)$.

Using Eqs. (36) and (37) and the values of $D$ and $H$ in Table 1, we can predict inverse dynamic moduli for the various experimental protocols. In Fig. 11 we show the predicted inverse dynamic moduli at $\tau=1 \mathrm{MPa}$, and $\sigma=5,10$, and $20 \mathrm{MPa}$. Each point in Fig. 11 is the first point of a line segment in $(\sigma, \tau)$ space, e.g. the $D$ and $H$ values derived for the $5 \theta 2$ loop are valid on a line from ( $5 \mathrm{MPa}, 1 \mathrm{MPa})$ to $(10 \mathrm{MPa}$, $8 \mathrm{MPa}$ ) (see Fig. 3). Furthermore, given Eqs. (39) and (40), we expect that the three lines of $K_{\text {dyn }}^{-1}$ derived for loops $5 \theta 2$,

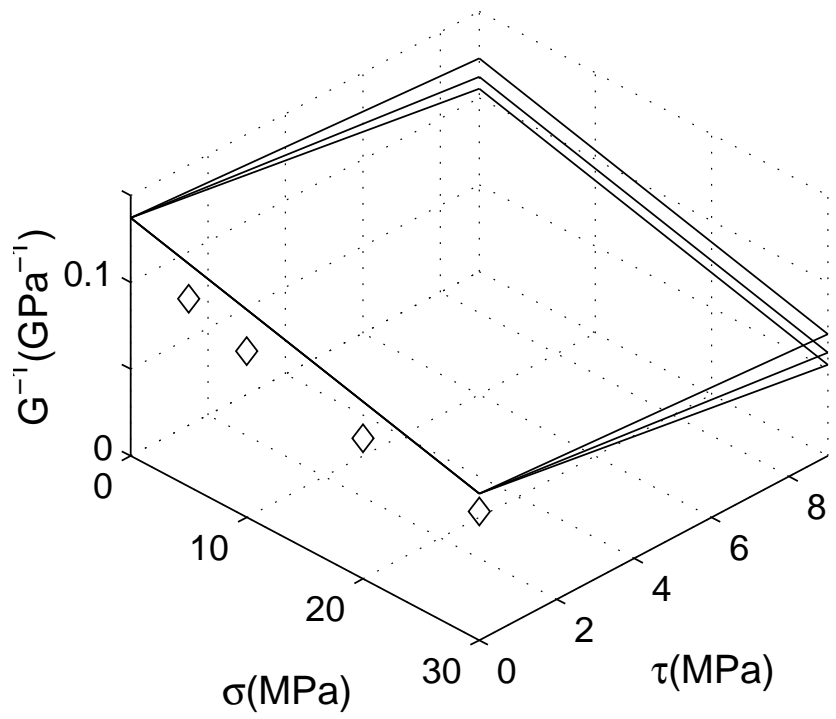

Fig. 13. Planes describing the inverse dynamic shear modulus. The top plane describes the modulus for constant $\sigma$, the middle plane is for constant $\theta$, and the bottom plane is for constant $\mu$. The diamonds are the result of dynamic measurements as a function of mean stress.

$10 \theta 2$, and $20 \theta 2$, will lie on a plane in $(\sigma, \tau)$ space. Because of the partial derivative $\partial \tau / \partial \sigma$ in Eq. (39), the inverse modulus plane derived for constant $\theta$ experimental protocols will be different from the plane derived for other experimental protocols. That is, we should find three planes of $K_{\text {dyn }}^{-1}$ and three planes of $G_{\text {dyn }}^{-1}$, one for each experimental protocol.

We performed a least squares fit of Eqs. (39) and (40) to the inverse dynamic modulus lines produced by Eqs. (36) and (37), where the fitting parameters were $K, G, A, B$, and $C$. Our results are $K=8.0 \mathrm{GPa}, G=7.3 \mathrm{GPa}, A=1900 \mathrm{GPa}$, $B=120 \mathrm{GPa}$, and $C=140 \mathrm{GPa}$. The values of $A, B$, and $C$ are not unreasonable, but are difficult to verify easily. Previous measurements of nonlinear elastic coefficients vary widely (Winkler and Liu, 1995). Figures 12 and 13 show the linear modulus planes described by our best fit parameters, and Eqs. (39) and (40). Also shown are results from time of flight measurements of moduli (Boitnott, 1997), where $\tau=0$ and the $\sigma=5,10,20 \mathrm{MPa}$ (equivalent mean stress). Our predictions are consistent with the time of flight modulus measurements, and quite close to the measurements of bulk modulus. The spread in the planes defined by the different protocols is also larger for the bulk modulus.

The analysis described here demonstrates the use of the PM space model to explore the relationship between nonlinear, hysteretic stress-strain measurements, and dynamic elastic measurements. The PM space model allows us to use quasistatic data to predict dynamic elastic properties of consolidated materials, and produces results consistent with independent measurements of dynamic quantities. In practice, this kind of measurement and analysis is time consuming and difficult, and is unlikely to be done routinely. Currently, the 
PM space model requires a closed data loop for the inversion procedure, i.e. creep cannot be accommodated. We are hopeful that a straightforward modification of the PM space model will allow us to account for creep. This would reduce the cycling necessary to obtain appropriate data. Meanwhile, to answer very specific questions, a limited test would suffice, i.e. a single experimental protocol.

Ideally, one would prefer to use dynamic measurements to infer quasistatic response, rather than the other way around. When an easy and reliable method for measuring third order elastic constants dynamically is developed, inverse modulus planes such as those in Figs. 12 and 13 will be trivial to derive. However, dynamic measurements will not so easily give information about the magnitude of the hysteresis $(\alpha)$. One can be reasonably sure that shear strain is more hysteretic than volumetric strain, but mechanisms for the hysteresis are still a matter of debate. As theory and experiment continue to complement each other, we hope to find a connection between dynamically measured quantities, and quasistatically displayed hysteresis.

Acknowledgements. The authors thank P. A. Johnson, J. A. TenCate, and K. E.-A. Van Den Abeele for continued interest and helpful discussions. This work has had partial support from the National Science Foundation, and the Institute of Geophysics and Planetary Physics at Los Alamos National Laboratory.

\section{References}

Boitnott, G. N.: Experimental characterization of the nonlinear rheology of rock, Int. J. Rock Mech. \& Min. Sci., 34, 379-388, 1997.

Guyer, R. A. and Johnson, P. A.: Nonlinear mesoscopic elasticity: Evidence for a new class of materials, Physics Today, 52, 30, 1999.

Guyer, R. A., Johnson, P. A., and TenCate, J. N.: Hysteresis and the dynamic elasticity of consolidated granular materials, Phys. Rev. Lett., 82, 3280-3283, 1999.

Guyer, R. A., McCall, K. R., Boitnott, G. N., Hilbert, Jr., L. B., and Plona, T. J.: Quantitative implementation of Preisach-Mayergoyz space to find static and dynamic elastic moduli in rock, J. Geophys. Res., 74, 5281-5293, 1997.

Holcomb, D. J.: Memory, relaxation, and microfracturing in dilatant rock. J. Geophys. Res., 86, 6235-6248, 1981.

Landau, L. D. and Lifshitz, E. M.: Theory of Elasticity, Pergamon, Tarrytown, N.Y., 1959.

Mayergoyz, J. D.: Hysteresis models from the mathematical and control theory points of view, J. Appl. Phys., 57, 3803-3805, 1985.

McCall, K. R. and Guyer, R. A.: A new theoretical paradigm to describe hysteresis, discrete memory and nonlinear elastic wave propagation in rock, Nonlin. Proc. in Geophys., 3, 89-101, 1996.

Preisach, F.: Über die magnetische Nachwirkung, Z. Phys., 94, 277, 1935.

Winkler, K. W. and Liu, X.: Measurements of third-order elastic constants in rocks, J. Acoust. Soc. Am., 98, 2886, 1995. 